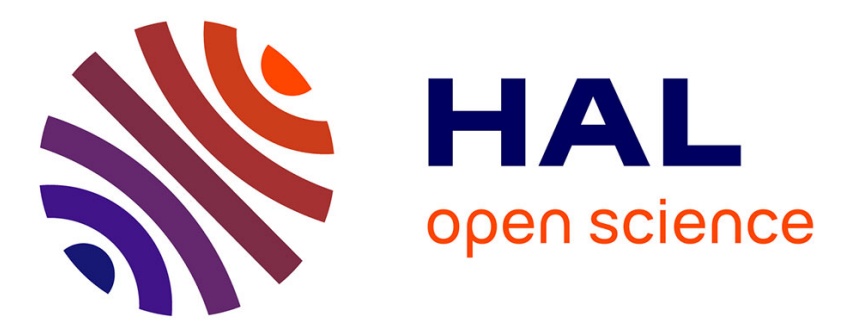

\title{
Révision d'une borne milliaire dédiée à Galère sur la via domitia (Castelnau-le-Lez, Hérault)
}

Bernard François, Christian Landes

\section{To cite this version:}

Bernard François, Christian Landes. Révision d'une borne milliaire dédiée à Galère sur la via domitia (Castelnau-le-Lez, Hérault). Gallia - Fouilles et monuments archéologiques en France métropolitaine, 1990, 47, pp.265-269. 10.3406/galia.1990.2912 . hal-01918324

\section{HAL Id: hal-01918324 \\ https://hal.science/hal-01918324}

Submitted on 20 Jan 2020

HAL is a multi-disciplinary open access archive for the deposit and dissemination of scientific research documents, whether they are published or not. The documents may come from teaching and research institutions in France or abroad, or from public or private research centers.
L'archive ouverte pluridisciplinaire HAL, est destinée au dépôt et à la diffusion de documents scientifiques de niveau recherche, publiés ou non, émanant des établissements d'enseignement et de recherche français ou étrangers, des laboratoires publics ou privés.

\section{(ㅇ)(1) $\$$}

Distributed under a Creative Commons Attribution - NonCommercial - NoDerivatives| 4.0 


\title{
RÉVISION D’UNE BORNE MILLIAIRE DÉDIÉE À GALÈRE SUR LA VIA DOMITIA (CASTELNAU-LE-LEZ, HÉRAULT)
}

\author{
par Bernard FRANÇOIS et Christian LANDES*
}

La restauration de cette borne milliaire apporte la preuve épigraphique du comptage des milles depuis Nimes jusqu'à Castelnau-le-Lez, l'antique Sextantio, située près de Montpellier (XXXI). Elle réfute l'hypothèse émise par Hirschfeld d'un bornage effectué depuis Narbonne.

Le monument, dédié au César Galère, constitue le reflet en Occident des victoires remportées par celui-ci sur les Parthes.

The restoration of that milestone gives epigraphic evidence of the counting of miles from Nimes to Castelnau-leLez, the antique Sextantio, located near Montpellier. It refules Hirschfeld's hypothesis of a counting from Narbonne.

The monument, dedicated to Galerius Caesar, is a reflection in the Western Empire of his victories over the Parthians.

Ce monument épigraphique fait partie du dépôt consenti par la Société archéologique de Montpellier au musée de Lattes ${ }^{1}$. Il fut découvert dans le lit du Lez près du moulin de Navitau au pied de l'oppidum de Sextantio, l'actuel Castelnau-le-Lez ${ }^{2}$. Les découvertes archéologiques des $\mathrm{xIX}^{\mathrm{e}}$ et $\mathrm{xx}^{\mathrm{e}} \mathrm{s}$. et les données de l'épigraphie tendent à relever l'importance de cette agglomération, signalée comme simple mutatio sur les itinéraires antiques, installée au

* Nous remercions $M^{\text {lle }}$ Émilienne Demougeot pour son aide et surtout Michel Christol qui a nous a encouragés à rédiger cette courte note et qui l'a attentivement relue.

1 Trente-sept objets parmi lesquels six bornes milliaires (CIL XII, 5654, 5651, 5661, 5622, 5653, 5663) qui jalonnaient le tracé de la voie domitienne entre Castelnau-le-Lez et Ambrussum. La borne étudiée appartient à cet ensemble (CIL XII, 5663) (musée de Lattes, inv. SAM 986 2).

2 M. P. Cazalis de Fondouce, Bornes milliaires du département de l'Ilérault, Mém. de la Soc. Arch. de Montpellier, VI, 1870-1876, p. 603; - A. Aurìs, Monographie des bornes milliaires du département du Gard, Mémoires de l'Académie du Gard, 1876, p. 228 ; - E. Bonnet, Antiquités et monuments de l'llérault, Montpellier, 1905 (rééd. Marseille, 1980), p. 94. franchissement du Lez par la voie domitienne ${ }^{3}$. Le séjour de la borne dans l'eau de la rivière explique la présence des concrétions calcaires qui la recouvraient partiellement. Aussi le texte, dès sa découverte fut-il publié avec des restitutions ou des lectures incomplè-

3 A l'oppidum protohistorique florissant succéda une agglomération qui dut en grande partie sa fortune au pont gallo-romain qui permettait à la voie domitienne de franchir le Lez. Les bases des piles qui le supportaient sont encore visibles au moment des basses eaux. Par la rivière et sa vallée, Sextantio était en relation avec le port de Lattes, implanté quelques kilomètres vers l'aval au débouché du Lez dans les étangs côtiers. La prospérité de l'ancien Castelnau-le-Lez est due à cette position de carrefour. Voir E. BonNeT, Antiquités et monuments du département de l'Héraull, Montpellier, 1905 (rééd. Marseille, 1980), p. 98, 118, 156, 207, 230; F. Daumas, R. Majurel, Rapport préliminaire sur les fouilles de Substantion, Gallia, 19, 1961, p. $12 ;-$ M. SAHUC, R. Ramonat, Sept tombes du secteur sud-ouest de la nécropole gallo-romaine de Navitau à Castelnau-le-Lez (Hérault), Archéologie en Languedoc, 1986, 1, p. 15 à 18. Parmi la dizaine d'inscriptions que le site a livrée, signalons CIL XII, 4189; voir également CIL XII, 4184, 4192, 4195, 4197 (aujourd'hui au musée de Lattes, inv. SAM 985 12), 4207, 4209, 4211 (musée de Lattes, inv. SAM 985 13), 4212, 4213. 


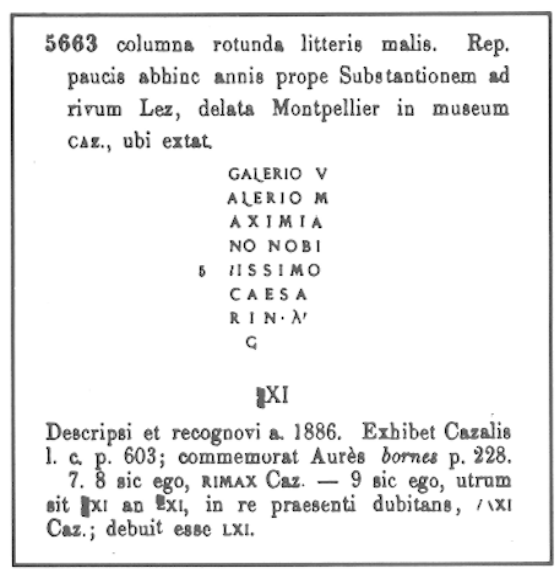

a

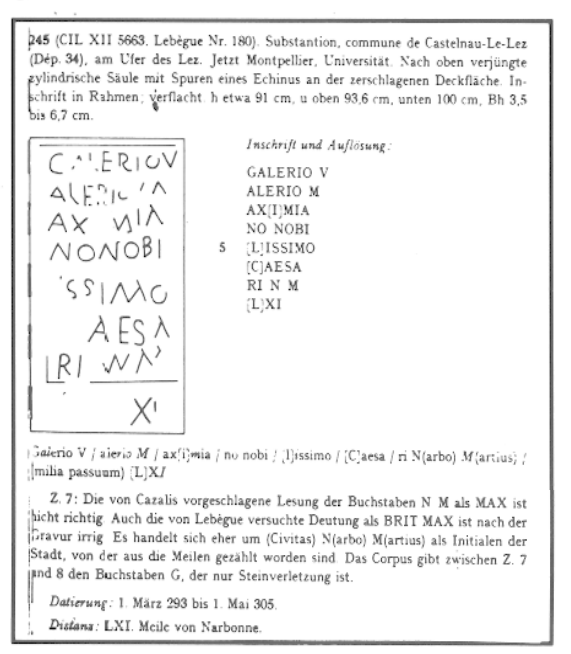

b

tes, qui ne furent jamais remises en question. C'est ainsi qu'il entre dans le tome XII du CIL, dans le livre sur les milliaires de Gaule Narbonnaise (Könnig, 1970), enfin dans le récent $C I L$ XVII, 2 (Berlin, 1986) (fig. 1). Récemment l'un de nous (B.F.) fut sollicité par la Mairie de Castelnau-le-Lez pour réaliser d'après l'original un moulage destiné à orner un espace public commémorant le passage de la voie. Le premier travail consista alors à enlever la gangue calcaire qui gênait l'opération*.

Le milliaire se présente sous la forme d'une petite colonne en calcaire coquillier miocène (type

4 Cette opération s'inscrit dans le programme de mise en valeur de la voie domitienne: voir A. Peyre, Une voie romaine en Languedoc : la via domitia, Le Courrier Archéologique du Languedoc-Roussillon, hiver 1985-86, 24, p. 1 à 27 ; A. Peyre, J. Lafforgue, Propositions de sauvegarde et d'aménagements de la via domitia, Les roules du sud de la France de l'Antiquité à l'époque contemporaine, $110^{\circ}$ Congrès National des Sociétés Savantes, Montpellier 1985, p. 61 à 77.

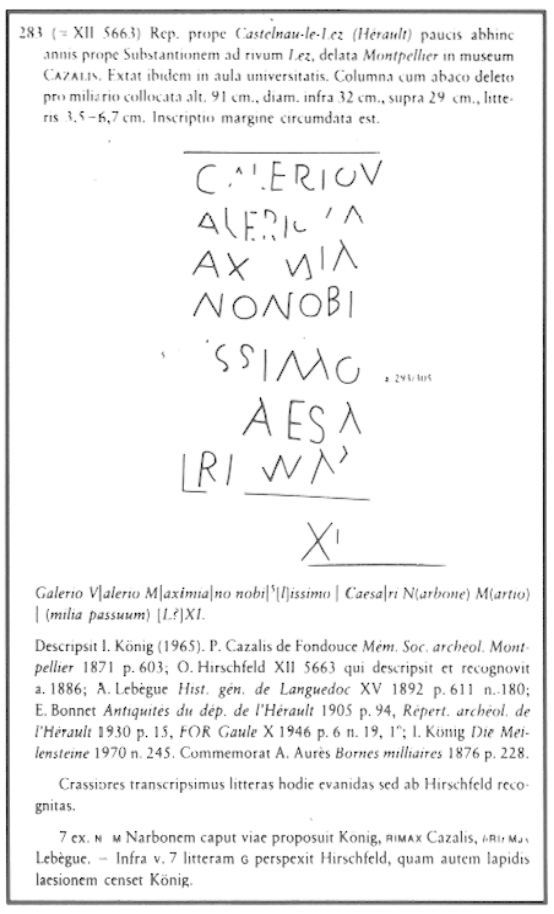

C

Fig. 1 - a, Hirschfeld, CII. XII 5663. b, König, Die Meilensteine der Gallia Narbonensis, Itinera romana, 3, Bern, $1970, \mathrm{n}^{\circ} 245$.

c, Walser, CIL XVII, 2, no 283.

"pierres de Castries»), d'environ $30 \mathrm{~cm}$ de diamètre ${ }^{5}$ et conservée sur $90 \mathrm{~cm}$ de hauteur. S'agit-il d'un réemploi comme c'est fréquemment le cas pour cette sorte de monument durant l'Antiquité tardive ${ }^{6}$ ? La nature même du matériau, assez impropre à la gravure, irait dans ce sens. Le champ épigraphique, haut de $67 \mathrm{~cm}$, large en moyenne de $33 \mathrm{~cm}$, est taillé en creux vers le tiers supérieur de sa hauteur. L'inscription se développe sur huit lignes (fig. 2) :

\section{GALERIO V/ALERIO M/AXIMIA/NO, NOBI/LISIMO/CAESA/RI. A N(emaus)O M(illia) $\mathrm{P}$ (assum) XXXI}

5 Diamètre maximal : $33,5 \mathrm{~cm}$; diamètre minimal : $29 \mathrm{~cm}$.

6 A. Grenier, Manuel d'archéologie gallo-romaine, $I I$. L'archéologie du sol. 1. Les routes, Paris, 1934 (rééd. 1985), p. $71 ;-M$. Labrousse, Un milliaire inédit de Constantin à Castelnau-Magnoac (Hautes-Pyrénées), Pallas, IV, 1956, p. 7073 ; - P. Sillitères, Les milliaires du sud de la péninsule ibérique, Epigraphie Hispanique, Paris, 1984, p. 271. 

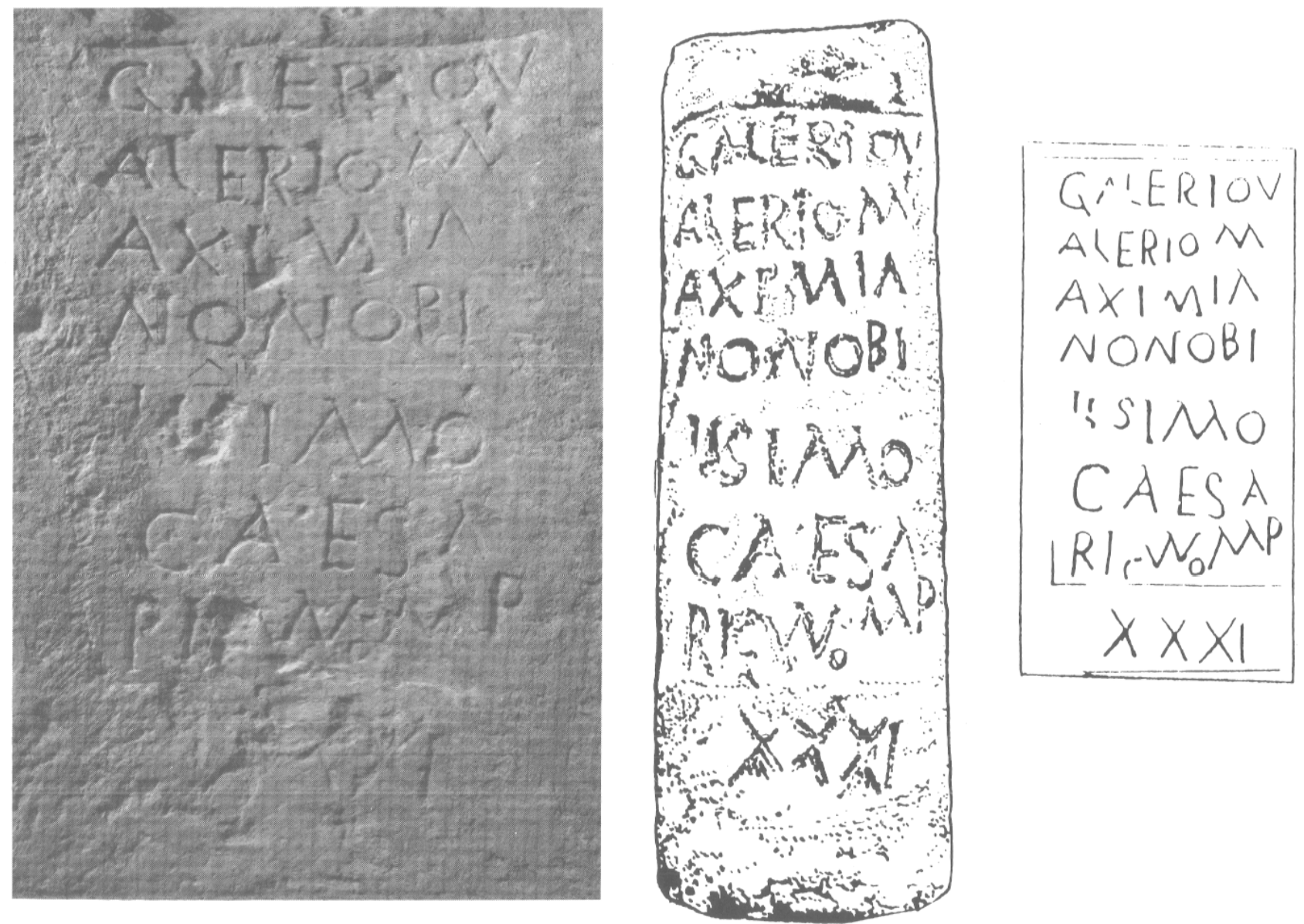

Fig. 2 - a, périphotographie de la borne après décapage, par procédé A. Chéné (GNRS, Centre Camille Jullian, Aix-en-Provence).

$b$, relevé de la borne après décapage.

A Galerius Valerius Maximianus, très noble César, De Nimes, $\mathrm{XXXI}^{\mathrm{e}}$ mille.

Il s'agit d'un "milliaire dédicace»" de la Tétrarchie dont la mise en page n'est pas parfaite. Les lignes ne sont qu'approximativement horizontales. Vers la droite, elles tendent à remonter en oblique, malgré deux traits de réglure (?) placés sous les lignes 7 et 8 . Les lettres sont de taille irrégulière ${ }^{8}$ : mais ce sont néanmoins des capitales classiques.

A remarquer : ligne 1, la petite barre oblique du $\mathrm{G}$ de Galerio qui dépasse largement vers le bas (influence de l'écriture cursive?); ligne 7, le 0

7 P. Siluı̀̀res, De la borne milliaire à la dédicace impériale. L'exemple de quelques inscriptions de l'Hispanie méridionale, Hommage à Robert Étienne, Paris, 1988, p. 357.

8 Ligne $1: 5,4$ à $3,6 \mathrm{~cm}$; ligne $2: 6$ à $4 \mathrm{~cm} ;$ ligne $3: 5$ à $4 \mathrm{~cm}$; ligne $4: 5$ à $4,5 \mathrm{~cm}$; ligne $5: 5$ à $4,5 \mathrm{~cm}$; ligne $6: 6,5$ à $5 \mathrm{~cm}$; ligne $7: 6$ à $2 \mathrm{~cm}$; ligne $8: 8$ à $6,5 \mathrm{~cm}$. minuscule de N(emaus)o, légèrement sous la ligne, comme surajouté ; ligne 8 , l'indication de la distance est détachée du reste du texte et les lettres sont plus hautes. On n'observe nulle part des points de séparation entre les mots pour faciliter la lecture.

Les six premières lignes ne laissent planer aucun doute sur l'identité du prince honoré : il s'agit de Galère, nommé César le $1^{\text {er }}$ mars 293 par Dioclétien, fondateur de la Tétrarchie, pour être son auxiliaire en Orient. Sa promotion à l'Augustat ( $1^{\mathrm{er}}$ mai 305$)$ permet de resserrer la fourchette chronologique dans laquelle se place notre inscription, entre le $1^{\text {er }}$ mars 293 et le 30 avril $305^{9}$. Aucune confusion n'est ici possible entre Galère et son neveu Maximin Daia. Tous deux possèdent le même double gentilice Galerius Valerius, mais leur surnom diffère par une

9 E. Stein, Histoire du Bas-Empire, 1, Paris, 1959, p. 68 . 
lettre, un $a$ nettement lisible à la fin de la ligne 3 : Maximia/nus pour Galère; Maximinus pour Maximin Daia ${ }^{10}$.

Mais c'est surtout sur l'indication de la distance que la restauration a apporté de nouvelles données corrigeant les hypothèses jusqu'ici présentées. König, suivant l'interprétation de Hirschfeld, proposait la lecture du LXI ${ }^{\mathrm{e}}$ mille depuis Narbonne. Récemment G. Walser, éditeur du CIL XVII, 2 (1986), se montrait plus réservé : [L ?] $\mathrm{XI}^{\mathrm{e}}$ mille. Or le décapage qu'a subi la borne permet d'être formel : elle indiquait aux voyageurs de la voie domitienne le $\mathrm{XXXI}^{\mathrm{e}}$ mille depuis Nimes. Il subsiste toutefois un problème; cette distance ne correspond pas exactement à celle que donnent les itinéraires antiques : XV milles de Sextantio à Ambrussum et XV milles supplémentaires d'Ambrussum à Nemausus (Nimes), soit XXX milles au lieu de XXXI'11. L'explication de cette anomalie se trouve à Ambrussum. En 1974, la découverte d'un tronçon de voie bien conservé prouvait que l'artère principale de la Province passait par l'intérieur de l'oppidum. Cette situation du Haut-Empire s'harmonise avec les distances mentionnées par les itinéraires. En revanche, au BasEmpire, vers la fin du $\operatorname{III}^{\mathrm{e}}$ et au début du IV ${ }^{\mathrm{e}}$ s., la voie domitienne ne serpentait plus sur la colline d'Ambrussum, mais la contournait vers le sud. Ce crochet doit correspondre au mille supplémentaire ${ }^{12}$. D'autre part, l'indication de l'origine du comptage des milles depuis Nimes renforce l'hypothèse du double bornage de la voie domitienne récemment formulée par Fr. Reynaud: depuis Narbonne, à l'époque julio-claudienne et depuis Nimes à partir du

10 P.-M. Duval, Un milliaire de Maximin Daia, Le Vieux Nimes, avril-juin, 1958, p. 1 ; - ID., Les inscriptions antiques de Paris, Paris, 1960, p. 113.

11 Gobelets de Vicarello, Auguste-Tibère; sur la date de ces quatre objets en forme de bornes milliaires, voir J. Heurgon, La date des gobelets de Vicarello, REA, 54, 1952, p. 45. Itinéraire d'Antonin, une compilation de la fin du $\mathrm{III}^{\mathrm{c}} \mathrm{s}$; l'Itinéraire de Bordeaux à Jérusalem, Ive s.; la Table de Peutinger en revanche montre une erreur de transcription à une époque indéterminée du Moyen Age du document d'origine ( ${ }^{\mathrm{e}} \mathrm{s}$.) : XX milles au lieu de XV entre Sextatione et Ambrussum : E. Desjardins, Géographie de la Gaule d'après la carte de Peutinger, Paris, 1869 (rééd. Bruxelles, 1969), p. 347; - G. Denizor, La voie domitienne entre Narbonne et le Rhône, Actes du 81* Congrès National des Sociètés Savantes; Paris 1968, p. 96-97; - J.-L. Fiches, Remarques sur le réseau antique dans la région de Nimes, Les routes $d u$ sud de la France de l'Antiquilé à l'époque contemporaine, op. cit., p. 136; - voir enfin le tableau récapitulatif de G. Walser, CIL XVII, 2, p. 76 .

12 J.-L. Fiches, Ambrussum et la voie domitienne, Revue d'Études Ligures, 1983, p. 152 et fig. 1, p. 136. règne d'Antonin ${ }^{13}$. Le milliaire de Castelnau-le-Lez prouve qu'à la fin du in ${ }^{e}$ s. et sur le territoire de la cité de Nimes tout au moins, les distances sont évaluées de Nimes vers Narbonne ${ }^{14}$. Or, en Narbonnaise, la mention du point de départ est exceptionnelle : outre la borne étudiée et celle de Maximin Daia trouvée aux environs de la maison carrée de Nimes, seul le milliaire de Constantin placé au $\mathrm{XIX}^{\mathrm{e}}$ mille de la c(ivitas) T(olosatum) sur l'axe Toulouse-Narbonne s'ajoute à cette courte liste ${ }^{\mathbf{1 5}}$. Sur l'inscription étudiée, le point de départ du bornage est marqué par $a$ et l'ablatif de la cité-ville de Nimes : a N(emaus) $0^{16}$. Ce n'est donc pas l'ensemble de la cité-peuple qui est ici considéré : dans cette hypothèse la formule aurait pu être $a c$ (ivitate) $N$ (emausensium) : elle figure ainsi sur le milliaire de Maximin Daia mentionné plus haut. La première expression, apparue en Gaule au II $^{\mathrm{e}} \mathrm{s}$. s'est maintenue durant le $\mathrm{II}^{\mathrm{e}} \mathrm{s}$., mais à la fin de cette période la référence aux cités-peuples est courante ${ }^{17}$ : a civ(itate) Par(isiorum) sur une borne trouvée à Paris ${ }^{18}$, a c(ivitate) $R$ (iedonum) à Rennes ${ }^{19}$ tandis qu'un milliaire de Vichy porte en toute lettre la mention de la civitas Arvernorum ${ }^{20}$.

Est-il anormal de trouver en Occident, dans les états administrés par le César Constance, des bornes érigées sous l'autorité des fonctionnaires impériaux par les cités chargées d'entretenir les routes et offertes à Galère, l'un des Tétrarques souverain d'Orient? Très souvent, certes, les Tétrarques y sont

13 Fr. Reynaud, La voie domitienne de Nimes à Castelnau-le-Lez, Etude topographique, lechnique et historique, Mémoire de maitrise, Univ. de Saint-Étienne, 1977, p. 95, 121 ; - Io., Le double bornage de la voie domitienne entre Nimes (Gard) et Castelnau-le-Lez (Ilérault), in: Mémoires II, Centre Jean Palerne, Univ. de Saint-Étienne, 1980, p. 102-121, cité par J.-L. Fiches, Ambrussum et la voie domitienne, op. cit., p. 135 et note 8.

14 Il en va de même pour le milliaire de Maximin Daia conservé à Nimes: Gal[erio]/Max[imi] no/nob(ilissimo) Caesari./C(ivitas) N(emausensium), daté entre le $1^{\text {er }}$ mai 305 et l'été 310, CIL XVII, 2, 243 (=CIL XII, 5624); König 205. Voir aussi E. Germer-Durand, Inscriptions antiques de $\mathrm{Ni}$ mes, Toulouse, 1893, no 82, p. 163.

15 A. Grenier, op. cit., p. 85.

16 Ibid., p. 86. Egalement en Orient: A Nicea mil(lia) $X X V I$ sur un milliaire dédié aux Tétrarques, Année Épigraphique (abrégé par la suite en A.E.), 1984, 832.

17 P.-M. Duval, op. cit., p. 119.

18 CIL XVII, 2, 494 (=CIL XIII, 8974).

19 CIL XVII, 2, 471 (= CIL XIII, 8953). Sur l'ethnique Riedones, A.-M. Rouanet-Liesenfelt, La civilisation des Riedones, Brest, 1980, p. 6-7.

20 CIL XVII, 2, 341 (=CIL XIII, 8905). 
associés $^{21}$ à l'image du célèbre groupe en porphyre conservé aujourd'hui à Venise. Cependant, on trouve trois autres bornes en Gaule dédiées au seul Galère ${ }^{22}$. Pourquoi un tel hommage rendu par Constance Chlore à son collègue d'Orient? Est-il lié au prestige retiré de la victoire obtenue sur les Perses par Galère en qui le rhéteur Eumène reconnaissait le symbole de

21 Comme le rappelle P. Silutikres, op. cit., p. 357. Signalons, parmi les milliaires de Gaule, ceux sur lesquels les Tétrarques sont réunis : CIL XVII, 2. 111, Martigny, Suisse (=CIL XII, 5520); CIL XVII, 2, 118. Monthey, Suisse; CIL XVII, 2, 120 b, Versuey, Suisse; CIL XVII, 2, 368, Amberac, Charente (=CIL XIII, 8914); CIL XVII, 2, 370, Sauvagnas (=CIL XIII, 8886); en Orient, A.E., 1978, 727 b; A.E., 1983, $905 ;$ A.E., 1984, 832. A remarquer également en Gaule des bornes dédiées aux deux seuls César, Constance Chlore et Galère : CIL XVII, 2, $13 \mathrm{~b}$, Digne $(=C I L$ XII, 5438) et CII XVII, 2, 90 a, Pontaix, Drôme (= A.E., 1963, 95).

22 S'ajoutent à la borne de Castelnau-le-Lez : CIL XVII, 2, 303, Baziège, Ilaute-Garonne (=CIL XII, 5676); CIL XVII, 2, 442, Cenon, Vienne (=CIL XIII, 8947); CIL XVII, 2, 620, Worms, RFA (=CIL XIII, 9087). En Afrique Proconsulaire, d'autre part, P. SAlAMA, Bornes milliaires d'Afrique Proconsulaire, Paris-Rome, 1987 (coll. de l'École Française de Rome, 101), p. 189. la domination romaine en Orient ${ }^{23}$ ? Cette hypothèse, si elle s'avérait exacte, nous permettrait de resserrer de quelques années le créneau chronologique dans lequel s'inscrit le milliaire de Castelnau-leLez, soit entre 298 et 305.

$\mathrm{Au}$ tournant des II I $^{\mathrm{e}}$ et IV $\mathrm{IV}^{\mathrm{e}} \mathrm{s}$., la voie domitienne qui relie presque en ligne droite Sextantio à Ambrussum est toujours entretenue. Les milles sont rigoureusement comptabilisés au bénéfice des voyageurs. La borne découverte à Castelnau-le-Lez constitue à la fois une dédicace en l'honneur de Galère mais témoigne aussi des réfections importantes dont cette artère majeure est encore l'objet à des fins probablement administrative (cursus publicus), économique et stratégique. Elle constitue un élément de la politique délibérée de rénovation des bornages et d'entretien des routes, admis par les historiens pour l'époque tétrarchique ${ }^{24}$.

Bernard Françors et Christian Landes

23 W. Seston, Dioclétien et la Tétrarchie, Paris, 1946, p. 180.

24 Voir en dernier lieu, P. Salama, op. cit., p. 238. 